\title{
New parameters for the analysis of statokinezigram vectors
}

\author{
Vladimir Ivanovich Usachev', Pierre-Marie Gagey², Viktor Egorovich Elyaev³, Anton Fedorovich Kononov³, \\ Grigory Anatolyevich Pereyslov ${ }^{3}$
}

\begin{abstract}
Based on the analysis of the statokinezigram vectors, new stabilometric parameters have been proposed: the Dynamic Stabilization Factor [DSF] and the Dynamic Stabilization Index [DSI]. They reflect the individual properties of the postural system and the functional state of the human body. DSI is stable in time and does not depend on the duration of the stabilometric recording. The principle and algorithm for calculating these parameters are presented. The possibility of evaluating the effectiveness of osteopathic treatment with its help is shown. Computer stabilometry was carried out on the stabilometric platform "Stabilan" with the software "StabMed" Special Design Bureau "Rhythm", Taganrog, Russia. The sampling rate was $50 \mathrm{~Hz}$.
\end{abstract}

Keywords: Stabilometry; Dynamic Stabilization; Statokinezigram Vectors.

\section{BACKGROUND}

Multi-minute recordings made with force platforms show slow postural oscillations of the body with a large amplitude and a one-minute period that cause a great variation in the classical parameters: "X-mean", "Y-mean", "Statokinezigram area", "Length of Statokinezigram", "LFS", "VFY" and "Romberg's Quotient"(1). It is therefore necessary to find parameters that are not disturbed by these one minute waves and do not depend on the duration of the recording. In seven-minute stabilometric recordings ${ }^{(2)}$, it was shown that the mean linear velocity (MLV) of displacement of the pressure center (COP) is more stable than the area of the statokinezigram (SKG). In addition, the area of the SKG in such a situation increases significantly $y^{(3)}$.

As an alternative to the usual SKG, T. Okuzono was proposed a vector-SKG ${ }^{(4)}$. Since 1996, we have proposed 12 stabilometric parameters based on the linear and angular velocities SKG vectors $^{(5)}$. In the process of verifying their informativeness in experiment and clinical practice, it was established that 9 of these parameters do not have clear advantages over classical stabilometric parameters. Three parameters turned out to be informative, but one of them, the accumulation function of the linear velocity of these vectors, which we called «The Quality of the Equilibrium Function» do not take into account the angular displacement of the vectors ${ }^{(6)}$. Therefore, we have developed two new parameters reflecting the dynamic stabilization of the body in an upright position ${ }^{(7)}$, which we represent here. They are called Dynamic Stabilization Factor (DSF) and Dynamic
Stabilization Index (DSI). The last parameter is calculated from the variance of the first and expressed as a percentage.

Synonyms of the equilibrium function are "balance», "stability of standing», "dynamic equilibrium», "dynamic stabilization". The term "dynamic stabilization" was proposed by P.-M.Gagey, M.Ouaknine, O.Sasaki ${ }^{(8)}$. It best reflects the physiology of the postural system, implying the unity and struggle of the processes of destabilization and stabilization of the body in an upright position.

\section{METHODS}

\section{The calculation of the DSF and DSI parameters Okuzono's Vector-SKG}

Okuzono considered SKG as a sequence of vectors, from one CoP position to the next, which are determined by the sampling frequency of the signal. Each vector characterized by its direction and its length in $\mathrm{mm}$ (Fig. 1).

All vectors are placed at the zero point of the Cartesian coordinate system. The prevalence of body deflection in the lateral or sagittal directions is assessed.

\section{Dynamic Stabilization Factor}

Placing the beginning of two successive vectors at the zero point of the coordinate system (Fig. 2), we see that vector $A B$, moving to the position of vector $B C$, forms the $A B C$ sector, which we called the "Dynamic Stabilization Factor" [8]. 


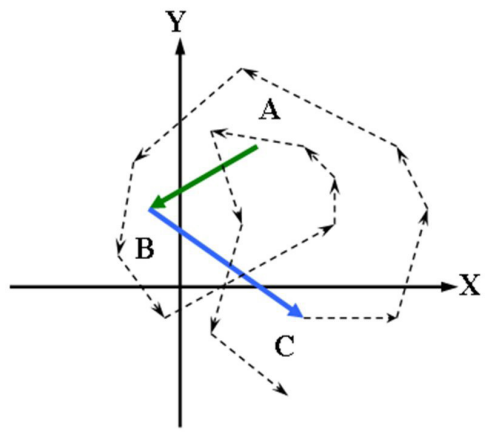

Figure 1. Previous vector $A B$ and the subsequent vector $B C$.

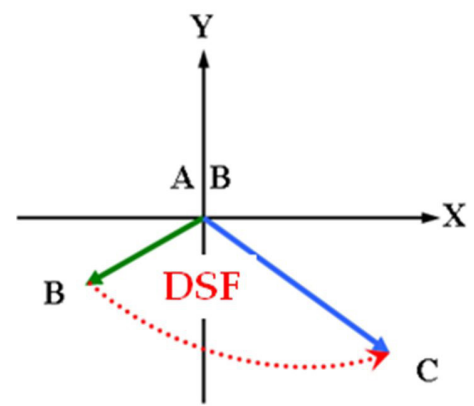

Figure 2. Vectors placed at the origin of the coordinate system. The displacement of the vector $A B$ to the vector $B C$ along the arc forms the sector $A B C$, called the DSF.

The sector area is calculated as swept out by the previous vector when moving to the next vector. When constructing the arc of the DSF sector, it is assumed that during the time of the change (20 ms at a sampling frequency of $50 \mathrm{~Hz}$ ), the length of the vector (linear velocity) and the angle vary uniformly with respect to time.

The instantaneous value of one DSF is given by the formula:

$$
D S F_{i}=\frac{\omega_{i}}{2} \cdot\left(V_{i}^{2}+V_{i} \cdot \Delta V_{i}+\frac{1}{3}\left(\Delta V_{i}\right)^{2}\right),
$$

Where:

$\omega_{i}$ is the angular velocity of the displacement of the subsequent vector (i+1) relative to the previous (i) in radians per second; $V_{i}$ is the linear speed $i$ of the vector in $\mathrm{mm} / \mathrm{sec}$;

$\Delta V_{i}=\left(V_{i+1}-V_{i}\right)$ is the difference between the linear speed $i+1$ of the vector and $i$ of the vector in $\mathrm{mm} / \mathrm{sec}$;

$V_{i+1}$ is the linear speed $i+1$ of the vector in $\mathrm{mm} / \mathrm{sec}$.

The dimension of the DSF is $(\mathrm{mm} / \mathrm{sec})^{2} \times$ radian $/ \mathrm{sec}$.

\section{Calculation of the Dynamic Stabilization Index}

The DSI, expressed as a percentage, is given by the formula:

$$
\mathrm{DSI}=100-\left(\log _{10} \mathrm{SD}_{\mathrm{DSF}}-2\right) / 0.04 \% \text {, }
$$

Where $\mathrm{SD}_{\mathrm{DSF}}$ is the standard deviation [SD] of DSF, calculated from the dispersion $[D]$ of all DSF values.

\section{Population}

477 healthy men aged 25 to 52 years were selected to compile a statistical description of the DSI. A per-minute comparison of the variability of the DSI with SKG area and MLV, was carried out with a seven-minute recording of 35 healthy men aged from 23 to 34 years. Clinical testing of the evaluation of the effectiveness of osteopathic treatment by DSF and DSI was performed in 238 patients. Of these, 162 women aged 18 to 47 years and 76 men aged 22 to 49 years. For example, the evolution of DSF and DSI after osteopathic treatment of one patient is presented.

\section{Statistics}

A statistical description of the DSI was performed using the Microsoft Office Excel Descriptive Statistics analysis package. The coefficients of variation of the SKG area, the average linear velocity of displacement of the COP and DSI in a seven-minute stabilometric record were also analyzed. The coefficient of variation (CV) of stabilometric parameters was calculated using the well-known mathematical formula:

$$
\mathrm{CV}=(\mathrm{SD} / \bar{X}) \times 100 \%,
$$

Where:

$\mathrm{CV}$ - coefficient of variation;

SD - standard deviation;

$\bar{X}$ - sample mean.

\section{Material}

Computer stabilometry was carried out on the stabilometric platform "Stabilan» with the software "StabMed» Special Design Bureau «Rhythm», Taganrog, Russia. The sampling rate was $50 \mathrm{~Hz}$.

\section{RESULTS}

\section{Descriptive statistics}

Table 1 presents the statistical description of the parameter DSI, among 477 healthy persons with open and closed eyes. Attention is drawn to the large interval of DSI values with open and closed eyes. The statistical significance of the difference between these two situations according to the Student's t-test is $p<0.001$.

\section{Comparation of DSI to SKG area and MLV}

We analyzed the coefficients of variation of the SKG area, mean linear velocity of displacement of the CoP and DSI in a seven-minute stabilometric recording. The SKG area variation coefficient was $36.38 \%$, the mean linear velocity of the CoP was $8.53 \%$ and the DSI was $0.85 \%$. This indicates the stability of the DSI, even in comparison with the average linear velocity of the CoP, not to mention the SKG area. 
Table 1 - Dynamic Stabilization Index parameter in normal subjects, in eyes open and closed situation.

\begin{tabular}{lcc}
\hline Statistical parameters & Eyes open & Eyes closed \\
\hline Average & 84.30 & 69.38 \\
Standard error & 0.49 & 0.80 \\
Median & 86.87 & 73.20 \\
Fashion & 93.51 & 86.70 \\
Standard deviation & 10.67 & 17.57 \\
Dispersion & 113.94 & 308.82 \\
Excess & 8.56 & 0.47 \\
Asymmetry & -2.27 & -0.90 \\
Interval & 83.95 & 94.82 \\
Minimum & 15.01 & 3.97 \\
Maximum & 98.96 & 98.79 \\
Number of persons & 477 & 477 \\
\hline
\end{tabular}

\section{DSF in various situations}

Along with DSI, the Dynamic Stabilization Factor dynamics can be used to evaluate the function of the postural system in various situations. In Fig. 3, 4 shows the difference in amplitude and instability of the DSF with open and closed eyes in 45 seconds at $f=50 \mathrm{~Hz}$.

With the eyes open, the DSF amplitude is small and only its "bursts» are observed, then with the eyes closed it increases significantly, and its «bursts» become frequent. This means that in the absence of visual information about the environment, managing equilibrium in the postural system becomes more complicated and the dynamic stabilization of the body deteriorates.

\section{Using the DSF and DSI to evaluate the effectiveness of treatment}

The osteopath can evaluate the effectiveness of his treatments thanks to the DSF and DSI ${ }^{(9)}$. For example, here is the result of a single session of general osteopathic treatment conducted by Christian Gerard, president of the Belgian Association of Osteopaths (fig. 5-7). These figures show graphs of the evolution of the DSF parameter over the entire recording time and give a unique DSI parameter value in percent.

Immediately after the osteopathic treatment, the DSF dispersion increased, and DSI decreased from $79 \%$ to $74 \%$ (Fig. 6). This indicated a worsening of the dynamic stabilization of the vertical position of the body. This situation is often observed immediately after a session of osteopathic treatment, which is associated with a reorganization in the body systems.

14 days after osteopathic treatment, the DSF dispersion became significantly less than the baseline level, and DSI increased from $79 \%$ to $86 \%$ (Fig. 7). This is explained to the classical canons of osteopathy, consisting in self-organization at a new level of body systems, including the postural system.

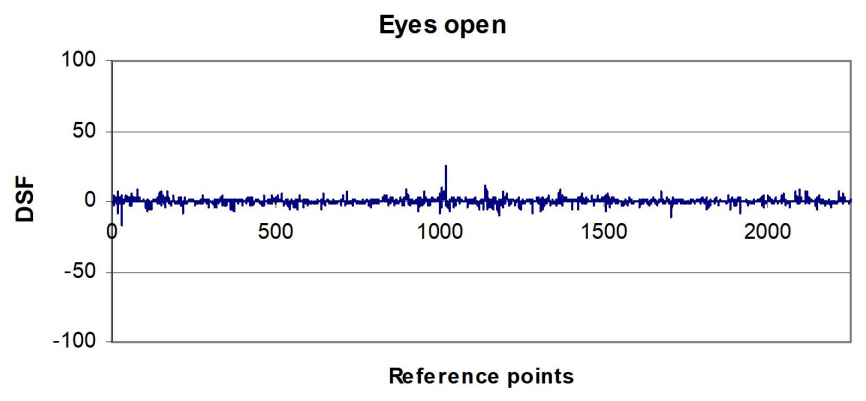

Figure 3. Dynamics of DSF healthy person with open eyes for 45 seconds at $f=50 \mathrm{~Hz}$.

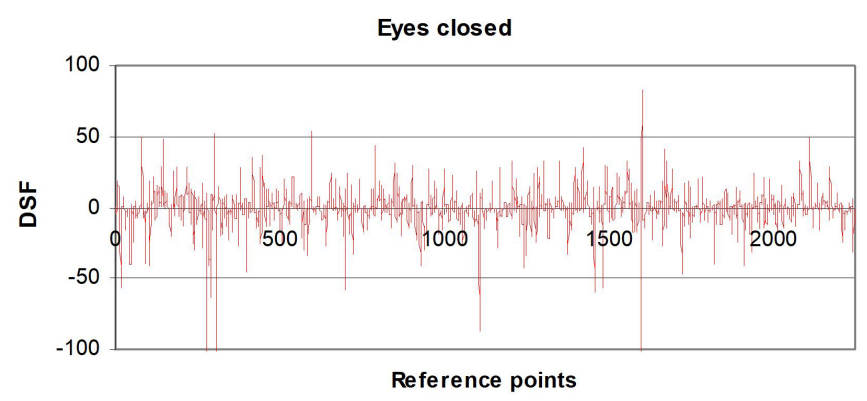

Figure 4. Dynamics of DSF healthy person with closed eyes for 45 seconds at $f=50 \mathrm{~Hz}$.

Dynamics of DSF before osteopathic treatment (DSI= $79 \%$ )

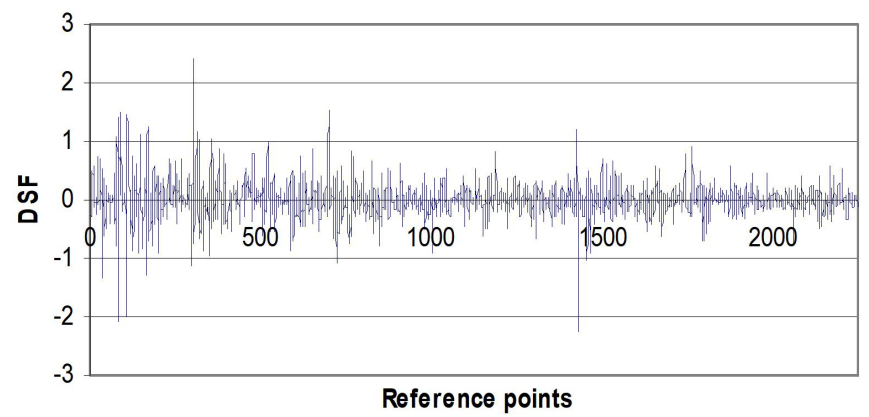

Figure 5. Dynamics of DSF and DSI value prior to osteopathic treatment in 45 seconds recording at $\mathrm{f}=50 \mathrm{~Hz}$.

\section{Dynamics of DSF after osteopathic treatment (DSI=74\%)}

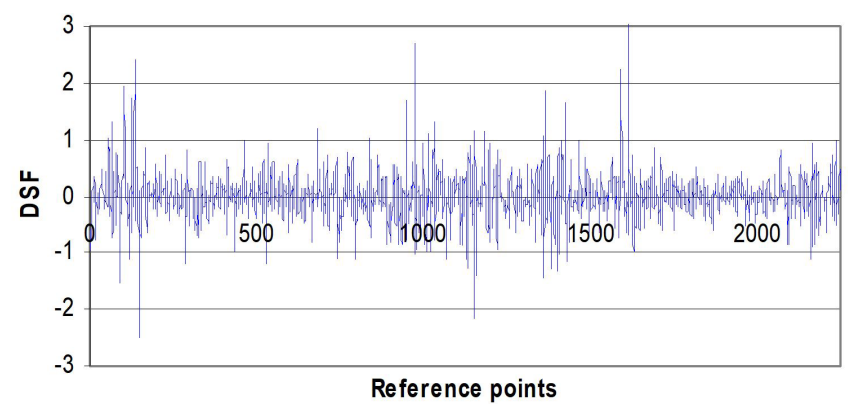

Figure 6. Dynamics of DSF and DSI value immediately after an osteopathic treatment session in 45 seconds recording at $\mathrm{f}=50 \mathrm{~Hz}$. 
Dynamics of DSF 14 days after treatment (DSI= $86 \%$ )

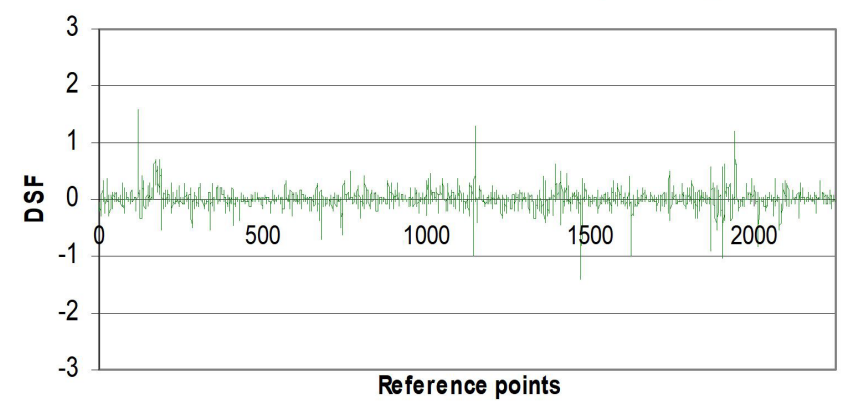

Figure 7. Dynamics of DSF and DSI value of the patient 14 days after osteopathic treatment in 45 seconds recording at $\mathrm{f}=50 \mathrm{~Hz}$.

\section{DISCUSSION}

The search for new stabilometric parameters led us two circumstances. This is the variability of classical parameters with slow postural oscillations and the opening of Okuzono statokinezigram vectors. The first showed the inconsistency of the traditional approach to the assessment of stabilometric information, and the second opened a new path in this direction. If Okuzono analyzed only the length of the statokinezigram vectors in $\mathrm{mm}$ and their direction, then we carried out a space-time analysis of vectors that takes into account the dynamics of the linear and angular velocity of the vectors and their integration.

We recognize that changes in the instantaneous values of linear and angular velocities CoP are random. However, the nature of the dynamics of DSF was informative. In various situations, for example, with open and closed eyes, the amplitude of its scatter and instability of values were significantly different. According to the graphs of the dynamics of DSF, you can easily distinguish one situation from another. It should be noted that when building the DSF sector arc, it is assumed that during the change (20 ms at a sampling frequency of $50 \mathrm{~Hz}$ ) the linear velocity and angle change uniformly with time. This method of calculation depends on the sampling rate of the signal. For frequencies of 40 and $50 \mathrm{~Hz}$, this is not significant, but at frequencies below $25 \mathrm{~Hz}$, a change in the shape of SKG is observed, which leads to a distortion of DSF values.

Since the main criterion for the difference is the variation of this parameter, in the mathematical expression is the variance, another parameter was proposed - the Dynamic Stabilization Index. DSI reflects the variance of all DSF during recording and is calculated as a percentage, which greatly simplifies its analysis. Statistical analysis of the DSI in the population shows two points. A wide range of individual differences and the statistical significance of the differences with open and closed eyes for each individual. It appears that the DSI reflects the individual genetically determined properties of the postural system, which change under different registration conditions. Analysis of the coefficients of variation SKG area, MLV and DSI with a seven-minute recording showed the smallest values of the coefficient of variation DSI. So DSI is stable in time and does not depend on the duration of the recording. This gives preference to this parameter in the diagnostic plan.
Using graphs of DSF dynamics and DSI values in percent, one can easily evaluate the effectiveness of osteopathic treatment. Thus, DSF and DSI, are new parameters for SKG vector analysis, can serve as informative parameters in stabilometric diagnostics.

\section{CONCLUSIONS}

1. Analysis of statokinezigram vectors is a promising direction for assessing the dynamic stabilization of the vertical position of the body.

2. The Dynamic Stabilization Factor reflects the space-time characteristics of the displacement of the pressure center.

3. The Dynamic Stabilization Index is stable over time and does not depend on the duration of the recording, which gives it preference over the SKG area and MLV.

4. DSF and DSI can serve as informative parameters in assessing the effectiveness of osteopathic treatment.

\section{AUTHORS CONTRIBUTIONS:}

VIU and VEB conducted the study; AFK carried out mathematical calculations of the stabilometric parameters; GAP conducted mathematical calculations and compiled a computer program; PMG helped to edit the article.

\section{CONFLICT OF INTEREST:}

nothing to declare.

\section{AUTHORS DETAILS}

2 Institute of Posturology, Paris, France;

${ }^{3}$ Closed Joint-Stock Company Special Design Bureau "Rhythm", Taganrog, Russia.

\section{REFERENCES}

1. Gagey PM. Introduction to the Russo-Japanese revolution in stabilometry: MTPRJ. 2018;16:584-585.

2. Usachev VI, Sliva SS, Belyaev VE. Stabilometric testing of a postural system- Proceedings of the XVIIth International Conference of ISPGR. International Society for Postural and Gait Research 2005;2:151.

3. Usachev VI, Belyaev VE. Evolution of SKG in time and space; 2017.

4. Okuzono T. Vector statokinesigram. A new method of analysis of human body sway: Pract. Otol. Kyoto.1983;10:2565-2580.

5. Usachev VI, Sliva SS, Belyaev VE, Pereyaslov GA, Pechorin PE. A new methodology for processing stabilometric information and the problems of its wide application in practice. Proceedings of the Conference Medical information systems. 2006:138-144.

6. Usachev VI. Estimation of dynamic stabilization of the center of pressure according to the analysis of vectors statokinezigramm. Publishing house St.-Petersburg Medical academy of post graduated education. 2008:291-296.

7. Usachev V, Gagey PM. Analyse vectorielle du statokinésigramme, estimation de la stabilisation dynamique de la position verticale du corps. Posturologie clinique. Tonus, posture et attitudes, Elsevier Masson. 2010:155-165.

8. Gagey PM, Ouaknine M, Sasaki O. Pour manifester la dynamique de la stabilisation. In: Lacour M. Posture et équilibre: Nouveautés conceptuelles, instrumentales et cliniques. Solal; Marseille. 2002:73-79.

9. Usachev VI. Estimation of dynamic stabilization of vertical body position in diagnostics of effectiveness of treatment and rehabilitation. Proceedings of the 5th International Posture Symposium. Bratislava: Academy of Sciences of Slovakia. 2008:53. 